\title{
DPSK Signals Demodulation Based on a Multimode Fiber with a Central Dip
}

\author{
Xiaoyong Chen, Paloma R. Horche, Alfredo Martín Minguez
}

\begin{abstract}
Differential Phase Shift Keying (DPSK) modulation format has been shown as a robust solution for next-generation optical transmission systems. One key device enabling such systems is a delay interferometer, converting the phase modulation signal into the intensity modulation signal to be detected by the photodiodes. Usually, a standard Mach-Zehnder interferometer (MZI) is used for demodulating a DPSK signal. In this paper, we develop an MZI which is based on all-fiber Multimode Interference (MI) structure: a multimode fiber (MMF) with a central dip, located between two single-mode fibers (SMFs) without any transition zones.

The MI based MZI (MI-MZI) is more stable than the standard MZI as the two arms share the same MMF, reducing the impact of the external effects, such as temperature and others. Performance of this MI-MZI is analyzed theoretically and experimentally from transmission spectrum. Experimental results shows that high interference extinction ratio is obtained, which is far higher than that obtained from a normal graded-index based MI-MZI. Finally, by software simulation, we demonstrate that our proposed MI-MZI can be used for demodulating a 40 Gbps DPSK signal. The performance of the MI-MZI based DPSK receiver is analyzed from the sensitivity. Simulation results show that sensitivity of the proposed receiver is about $-22.3 \mathrm{dBm}$ for a BER of $10^{-15}$ and about $-23.8 \mathrm{dBm}$ for a BER of $10^{-9}$.
\end{abstract}

Keywords-optical communication; differential phase shift keying; modal interference; optical reciever

\section{INTRODUCTION}

Differential Phase Shift Keying (DPSK) is an attractive modulation format for next-generation optical transmission systems since it exhibits high tolerance to chromatic dispersion and robustness to nonlinear effects. Besides these advantages, it also offers an improved receiver sensitivity by balanced detection. In DPSK communications, one key device enabling to convert the differential phase information into intensity modulation information to be detected by the photodiodes is a delay interferometer, such as Mach-Zehnder interferometer (MZI). Usually, MZI is used for demodulating DPSK signals because of its simple structure. In recent years, allfiber MZI is proposed to take place of the standard MZI for improving the performance. It is not only used for measurement of temperature, pressure, flatness of plane optical plates, thickness of thin film, coherence length of a laser [1-2], but also used in optical communication systems, such as DPSK demodulator [3-6]. However, in the DPSK demodulation scheme proposed in [3], there is one problem of stability due to the two beams go through two different paths. For further improving the performance of all-fiber MZI, several schemes are proposed recently. In [4], a MZI based on a special step-index multimode fiber (MMF) is proposed to demodulate a $10 \mathrm{Gbps}$ DPSK signal. In [5], a photonic crystal fiber based MZI for DPSK signal demodulation is presented. In this scheme the device is fabricated by mismatch splicing a PCF between two standard single mode fibers (SMFs).

All-fiber based MZI is appealing due to its properties of low-cost, simple-design and easy-implementation. In this paper we propose an all-fiber Multimode Interferencebased MZI (MI-MZI) based on a MMF with a central dip, for DPSK signals demodulation. Compared with the schemes proposed in [4] and [5], our proposed scheme not only reduces the cost, but also improves the receiver performance.

In our previous work, a MI-MZI device has been investigated for demodulating a $10 \mathrm{Gbps}$ DPSK signal [6]. In this work we further study the application for demodulating a 40 Gbps DPSK signal. Power distribution in the MMF is studied and the impact of interference extinction ratio of MI-MZI on the receiver performance is also discussed in this paper. The paper is organized as follows. The theory of MI-MZI is presented in Section II.

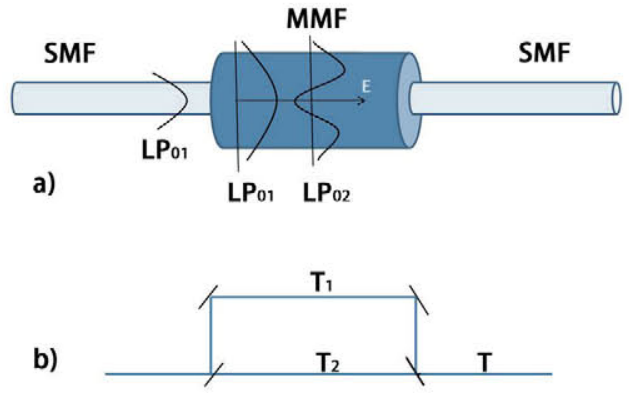

Fig. 1. Comparison between MI-MZI structure (a) and a standard MZI (b) 
Experimental results are shown and discussed in Section III. In Section IV we present the simulation structure for demodulating DPSK signals and discuss the simulation results. Finally, the conclusions are presented in section V.

\section{THEORY}

The modal interference process is a well known phenomenon and it had been studied by many authors [712]. Based on this principle we can make an interferometer as a MZI. The structure of the MI-MZI is shown in Fig. 1(a) where only core fibers are drawn. The structure is composed of a multimode region located between two single-mode regions without any transition zones. This passive device has been made with just a multimode fiber $(50 / 125 \mu \mathrm{m})$ spliced between two single-mode fibers $(9 / 125 \mu \mathrm{m})$. Moreover, a simple connector (with a matched liquid index to avoid reflection interfaces) can be employed to couple single-mode and multimode fibers without any fusion. Both configurations have been employed by us and similar results have been obtained $[8$, 10]. Compared with the standard MZI, as shown in Fig. 1(b), which obtains a time delay through the difference of the two physical paths, the MI-MZI obtains a time delay from time difference for both modes passing through the MMF transmission path. This can reduce the effect caused by the external environment, such as temperature, and improve its stability.

Assuming that the SMF and the MMF are ideally aligned, due to the circular symmetry of the input field, only the symmetrical modes $L P_{0 k}$ will be excited in the $\mathrm{MMF}$ when light travels from SMF to MMF. These modes propagate with different propagation constants and they will interfere with each other along the direction of propagation. The light power coupled into the SMF-output fiber depends on the relative phase difference at the end of the multimode fiber $(L)$. According to $[1,2]$, the power coupled into the second SMF can be written as:

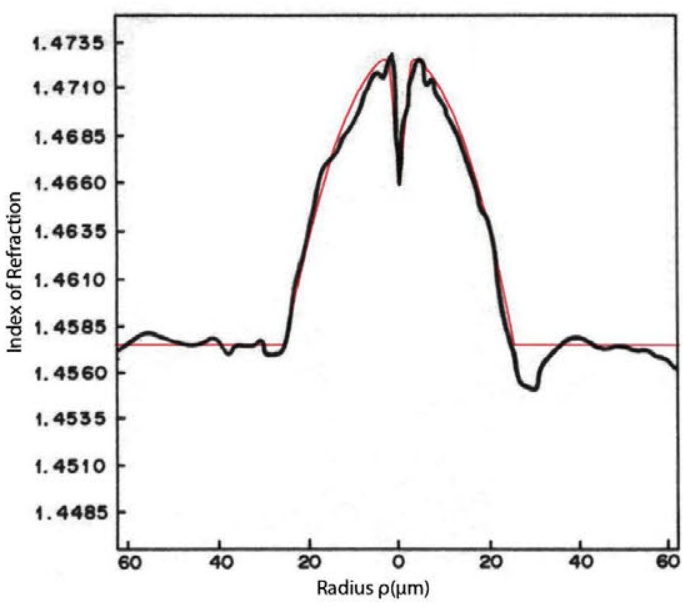

Fig. 2. Refractive index profile of the MMF with a central dip: the red line is the model and the black line is the measured profile

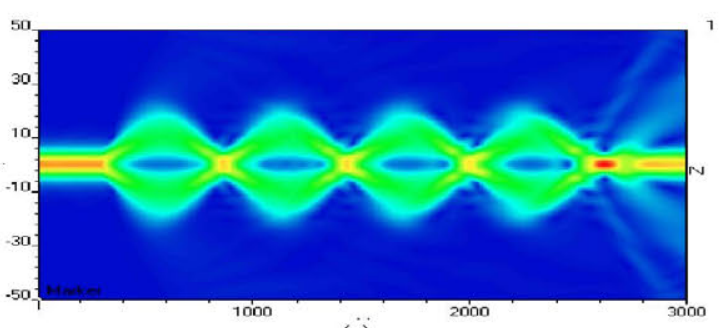

(a)

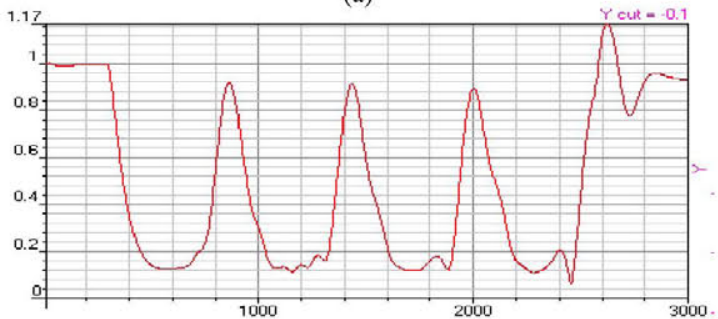

(b)

Fig. 3. (a) Power distribution of the light inside the graded-index MMF with a central dip. (b) Power along the optical axis of the MMF.

$$
\begin{aligned}
\mathrm{P}_{\text {out }}=\left|\int_{0}^{\infty} \psi(r, L) E_{\text {in }}^{*} r d r\right|^{2} \\
=\left|\sum_{\mathrm{k}=1}^{\mathrm{K}} c_{k}^{2} \exp \left(\mathrm{i}\left(\beta_{1}-\beta_{k}\right) L\right)\right|^{2}
\end{aligned}
$$

where $E_{\text {in }}$ is the normalized input field and $\psi(r, L)$ are the the normalized eigenmodes which are determined by the refractive profile of the $\mathrm{MMF} . c_{k}$ is the coupling coefficient between the input mode and the excited mode. $\beta_{k}$ is the propagation constant of each excited mode. The MMF we studied in this work is the graded-index with a central dip, as shown in Fig. 2. According to (1), we can calculate the power distribution along the propagation direction, as shown in Fig. 3.

Although many modes will be excited and propagate in the MMF, most of the power is coupled into the first two circular symmetrical modes $L P_{01}$ and $L P_{02}$ since these two modes have similar azimuthal symmetry and the phase mismatch are minimized. In comparison with the gradedindex $\mathrm{MMF}$ and step-index MMF, graded-index $\mathrm{MMF}$ with a central dip will reduce the input power coupled into the excited fundamental mode $L P_{01}$ and increase power coupled into the second circular symmetrical mode $L P_{02}$. This effect will increase the interference extinction ratio, making the device working as a quasi-MZI. Although some power is still coupled into other high-order modes, these modes will not seriously affect the interference between modes $L P_{01}$ and $L P_{02}$. The transmission is periodic with respect to the frequency and is usually represented by a comb-filtering spectrum as depicted, i.e., in Fig. 5. The spacing $\Delta \lambda$ between the adjacent constructive peaks (destructive valleys) can be written as [13]:

$$
\Delta \lambda=\frac{\lambda^{2}}{\left|n_{01}-n_{02}\right| L}=\frac{2 \pi \lambda}{\left|\beta_{01}-\beta_{02}\right| L}=\frac{\lambda z_{b}}{L}
$$


where $n_{01}$ and $n_{02}$ are the effective index of modes $L P_{01}$ and $L P_{02}$, respectively. $z_{b}=\frac{2 \pi}{\left|\beta_{01}-\beta_{02}\right|}$ is the beat length of the MMF [14]. From (2) we can see that it is possible to adjust the separation between peaks by changing the length of the MMF.

The difference of propagation constants of the two beating modes can be written as:

$$
\beta_{01}-\beta_{02}=\frac{\lambda}{4 \pi a^{2} n_{\text {core }}}\left(U_{01}^{2}-U_{02}^{2}\right)
$$

with

$$
\begin{aligned}
& \mathrm{U}_{01}=2.405 \mathrm{e}^{-1 / \mathrm{v}} \\
& \mathrm{U}_{02}=5.520 \mathrm{e}^{-1 / \mathrm{V}} \\
& V=\frac{2 \pi a}{\lambda} \sqrt{n_{\text {core }}^{2}-n_{\text {clad }}^{2}}
\end{aligned}
$$

where $a$ is the core radius, $\lambda$ is the wavelength in vacuum, $n_{\text {core }}$ is the maximum refractive index of the core and $n_{\text {clad }}$ is the refractive index of the cladding.

The transmission spectrum of this MI-MZI can be described as follows:

$$
\begin{gathered}
T=T_{1}+T_{2}+2 \sqrt{T_{1} T_{2}} \cos (\varphi) \\
\varphi=\int_{0}^{L} \Delta \beta(z) d z=L \Delta \beta_{12}
\end{gathered}
$$

where $T_{1}$ and $T_{2}$ are the intensity of the two modes $L P_{01}$ and $L P_{02}$, respectively. $L$ is the length of the MMF. $\Delta \beta_{12}$ is the difference of propagation constants of modes $L P_{01}$ and

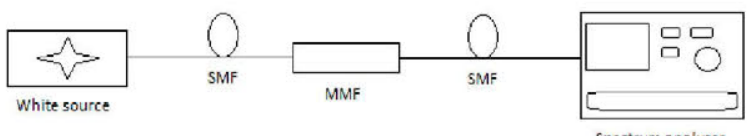

Fig. 4. Experimental setup

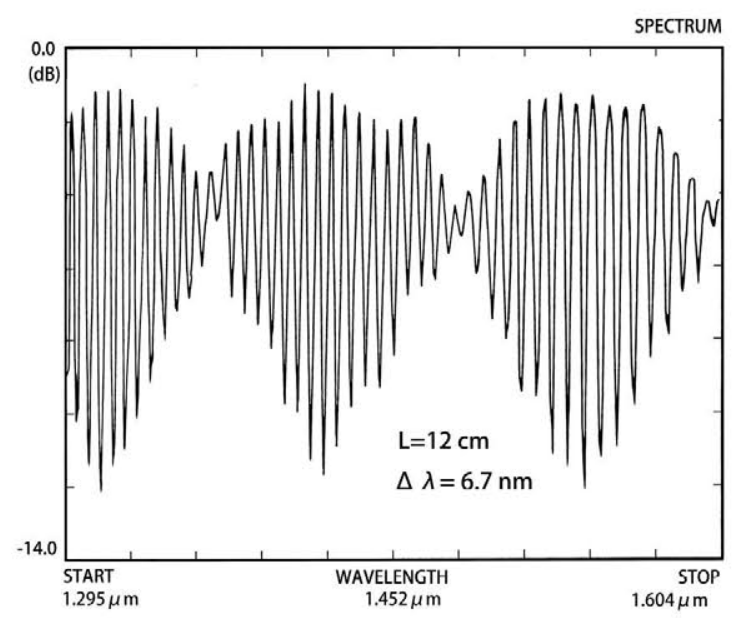

Fig. 5. (a) Transmission spectrum of MI-MZI with MMF of $12 \mathrm{~cm}$

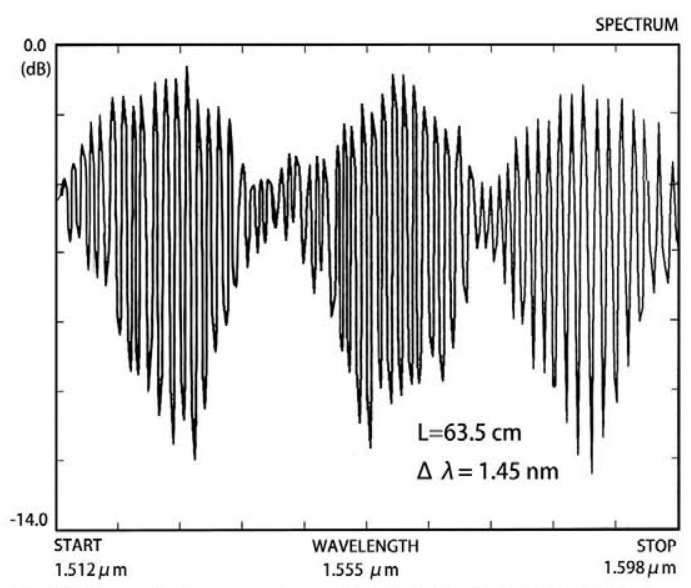

Fig. 5. (b) Transmission spectrum of MI-MZI with MMF of $63.5 \mathrm{~cm}$

$L P_{02}$. Combining with the (2), the time delay between two modes can be defined by the following function:

$$
\Delta t=\frac{L}{c / n_{01}}-\frac{L}{c / n_{02}}=\frac{\Delta n L}{c}=\frac{\lambda^{2}}{c \Delta \lambda}
$$

Here we define another parameter which is called delay coefficient $\Delta d$. It is corresponding to the time delay in a one-meter MMF. It can be described as:

$$
\Delta d=\frac{\Delta t}{L}=\frac{\Delta n}{c}=\frac{\lambda^{2}}{c L \Delta \lambda}
$$

We can see that the delay coefficient of the MI-MZI is only determined by the effective index difference $\Delta n$ between two modes, which is governed by the MMF profile.

\section{EXPERIMENTAL RESULTS}

The experimental setup used for measuring the transmission spectrum is shown in Fig. 3. In the experiment a white light is used as the light source since it has a very wide spectrum from $0.4 \mu \mathrm{m}$ to $1.62 \mu \mathrm{m}$. The $\mathrm{MMF}$ has a profile as shown in Fig. 2. Its diameters of core and cladding are $50 \mu \mathrm{m}$ and $125 \mu \mathrm{m}$, respectively. The maximum refractive index of core is 1.473 and the clad is 1.4567. Finally, a spectrum analyzer is used to observe and record the transmission spectrum.

In order to observe the spacing $\Delta \lambda$ between adjacent constructive peaks (destructive valleys) changing with the length of MMF, we use two different lengths of MMFs (12 $\mathrm{cm}$ and $63.5 \mathrm{~cm}$ ). The experimental results are shown in Fig. 5. It can be seen that increasing the length of MMF from $12 \mathrm{~cm}$ to $63.5 \mathrm{~cm}$ leads to a decreasing of spacing $\Delta \lambda$ from $6.7 \mathrm{~nm}$ to $1.45 \mathrm{~nm}$. These results are in agreement with the theory which requires that the spectral period should be inversely proportional to the length of the MMF. Fig. 5 also shows that the outline of the transmission spectrum oscillates with the frequency. This indicates that some high-order modes are excited and propagating in the MMF. This result also agrees with the theory. According to (7), it can be calculated that the delay coefficient of the 
$\mathrm{MMF}$ is $8.7 \mathrm{ps} / \mathrm{m}$, which is far higher than that $(3.48 \mathrm{ps} / \mathrm{m})$ of the step-index MMF used in [4].

\section{MI-MZI FOR DPSK SIGNALS DEMODULATION}

Considering the MI-MZI as a delay line interferometer (DI), it can be directly used for demodulating DPSK signals. The schematic diagram of MI-MZI for DPSK signals demodulation is shown in Fig. 6. We follow it to build a simulation system.

In order to observe the relationship between the receiver's sensitivity and the interference extinction ratio of the proposed MI-MZI, a tunable laser is used in the simulation. A Mach-Zehnder modulator (MZM), which is driven by a 40 Gbps DPSK data stream, is used to modulate the light to generate a 40 Gbps optical NRZDPSK signal. The MMF used in the simulation has the profile as shown in Fig. 2 (red line). It can be calculated that the length required for generating $25 \mathrm{ps}$ time delay between two modes is $2.89 \mathrm{~m}$. The transmission spectrum of MI-MZI with MMF of $2.89 \mathrm{~m}$ is shown in Fig. 7. Finally, a photodiode is used to detect the demodulated signal and a bit error rate (BER) analyzer is used to analyze the performance of the received signal.

From the transmission we can calculate that the spacing $\Delta \lambda$ is $0.33 \mathrm{~nm}$ and the delay coefficient of this fiber model is $8.3 \mathrm{ps} / \mathrm{m}$. These values are very close to the results got from the experiment. It demonstrates that this fiber model is the right model for taking place of the real fiber. Thus, the simulated results based on this fiber model have significant meaning to the real conditions.

The simulation results are shown in Fig. 8. The inset of eye diagram shows a relatively open eye which can provide error-free operation. The sensitivity of our proposed receiver in wavelength of $1550 \mathrm{~nm}$ is about -22.3 $\mathrm{dBm}$ at a BER of $10^{-15}$ and about $-23.8 \mathrm{dBm}$ at a BER of $10^{-9}$. Compared with the typical DI DPSK receiver [15], which is built with two fiber couplers, our proposed receiver has about $0.6 \pm 0.2 \mathrm{~dB}$ power penalty. This penalty is caused by several aspects, such as the loss in the $\mathrm{MMF}$, and the low interference extinction ratio between the two modes. The interference extinction ratio depends on two factors. One is the power difference of the two beating modes. From (4) we know that the power difference is smaller, the interference extinction ratio is higher. The other factor is other high-order modes are excited and propagating in the MMF. Although the gradedindex MMF with a central dip will reduce power difference between two beating modes, some other high-order modes, such as $L P_{03}$ and $L P_{04}$, are also excited and propagating in the MMF.

The simulation results also show that the sensitivity will be aggravated with the interference extinction ratio decrease. The power penalty is more than $3 \mathrm{~dB}$ when the extinction ratio is lower than $4 \mathrm{~dB}$. This is the limitation of the MI-MZI based receiver.

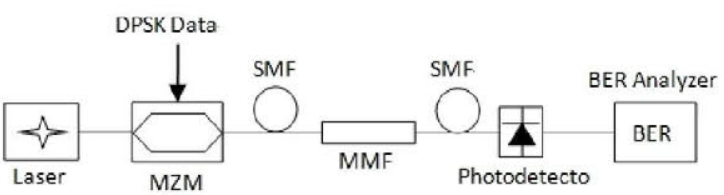

Fig. 6. Stucture of MI-MZI for DPSK signals demodulation. MZM: Mach-Zehnder modulator; BER: bit error rate

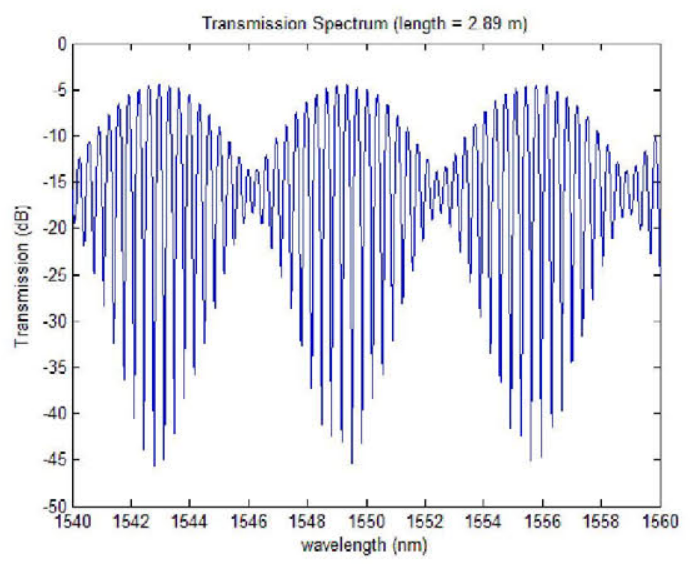

Fig. 7. Transmission spectrum of MI-MZI with length of $2.89 \mathrm{~m}$.

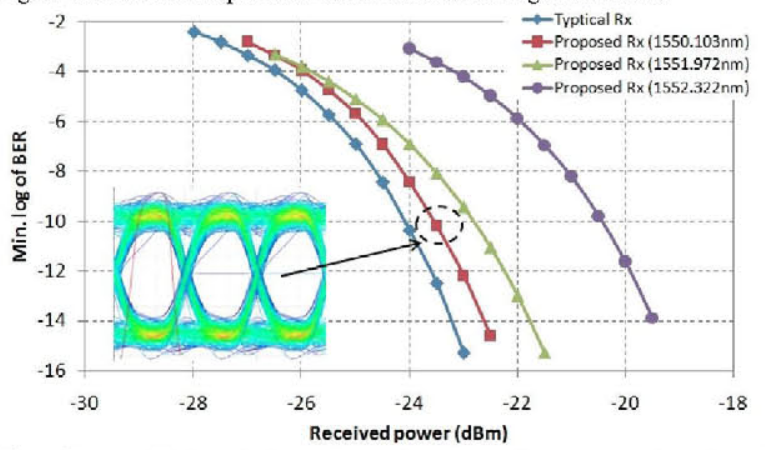

Fig. 8. Sensitivity of the MI-MZI based receiver at wavelengths of $1550.103 \mathrm{~nm}, 1551.972 \mathrm{~nm}$ and $1552.322 \mathrm{~nm}$ by compared with the typical receiver. The inset is the eye diagram.

\section{CONCLUSION}

We have demonstrated a MI-MZI, which is based on a graded-index MMF with a central dip between two SMFs. In this structure the excited fundamental $\left(L P_{01}\right)$ and first order symmetry $\left(L P_{02}\right)$ modes are propagating in the $\mathrm{MMF}$ with different phase constants. These two modes interfere with each other along the direction of propagation and the light power coupled into the SMF-output fiber depends on the relative phase difference at the end of the MMF. Thus, we can obtain an interference pattern at the exit of the second SMF. The experimental results are in agreement with the theory and a delay coefficient of $8.7 \mathrm{ps} / \mathrm{m}$ is obtained. Finally, a simulation of using the MI-MZI to demodulate a DPSK signal is done. The simulation results show that MI-MZI based DPSK receiver has similar performance as the typical DI receiver, but with about 0.6 $\pm 0.2 \mathrm{~dB}$ power penalty at wavelength of $1550 \mathrm{~nm}$. 
However, compared with the all-fiber MZI based DPSK receiver proposed in [4] and [5], our receiver not only cost less, but also shows better performance.

Since the design of MI-MZI is low cost and easily manufactured, it will be used widely in many optical applications in the future, such as using as filters or temperature sensors. Moreover, with the proper design it also can be used as WDM multiplex and demultiplex.

\section{ACKNOWLEDGMENT}

The authors would like to acknowledge support from the China Scholarship Council (CSC).

\section{REFERENCES}

[1] Q. Wang, G. Farrell, and W. Yan, "Investigation on Single-ModeMultimode-Single-Mode Fiber Structure," J. Lightwave Technology, Vol. 26, No. 5, 512-519, 2008

[2] M. Kumar, A. Kumar and S. M. Tripathi, "Acomparison of temperature sensing characteristics of SMS structures using step and graded index multimode fibers," Optics Communications, Vol. 312, pp. 222-226, 2014

[3] F. Séguin, and F. Gonthier, "Tuneable All-Fiber Delay-Line Interferometer for DPSK Demodulation," in Tech. Dig. OFC'2005, pp. OFL 5, March 2005

[4] Y. K. Lize, R. Gomma and R. Kashyap, "Low-cost multimode fiber Mach-Zehnder interferometer for differential phase demodulation", in proc. SPIE 2006, San Diego, USA, August 2006

[5] J. Du, Y. Dai, G. K. P. Lei, W. Tong, and C. Shu, "Photonic crystal fiber based Mach-Zehnder interferometer for DPSK signal demodulation", Optics Express, Vol. 18, No. 8, pp. 7917-7922, 2010
[6] X. Chen, D. Garcia, A. M. Minguez, and P. R. Horche, "MachZehnder Interferometer Based on All-fiber Multimode Interference Device for DPSK Signal Demodulation", in Proc. URSI 2013, Santiago de Compostela, pp. 161, September 2013

[7] M. Blahut and D. Kasprzak, "Multimode interference structures properties and applications", Optica Applicata, Vol. XXXIV, No. 4 pp. 573-587, 2004

[8] P. R. Horche, M. A. Muriel, J. A. Martin-pereda, "Measurement of Transmitted Power in Untapered Multifibre Unions: Oscillatory Spectral Behaviour", Electronics Letters, Vol. 25, No. 13, pp. 843844, 1989

[9] P. R. Horche, "An Optical Multi-Bandpass Filter Obtained by Combining Bragg Reflection, Intermodal Coupling and Interference Process in an All-Fibre Device". In proc. NOC 1998, pp. 186-189 1998

[10] P. R. Horche, "Measurement of the oscillatory spectral behavior of abruptly dissimilar unions fabricated with dispersion shifted fibre: Filter Application", in proc. NOC 1996, pp. 314-315, 1996

[11] K. Abe, Y. Lacroix, L. Bonnell, and Z. Jakubezyk, "Modal Interference in a Short Fiber Section: Fiber Length, Splice Loss, Cutoff, and Wavelength Dependences", Journal of lightwave technology, VOL. 10, NO. 4, pp; 401-406,1992

[12] M. N. Petrovich, F. Poletti, D. J. Richardson, "Analysis of Modal Interference in Photonic Bandgap Fibres", in proc. ICTON 2010, pp. Tu.B2.3, 2010

[13] P. R. Horche, M. López-Amo, M. A. Muriel and J. A. MartinPereda, "Spectral Behavior of a Low-Cost All-Fiber Component Based on Untapered Multifiber Unions". IEEE Photonics Technology Letters, Vol 1, No 7, pp. 184-187,1989

[14] P. R. Horche, "Transmissions Propoerties of All-Fibre Modal Interference Devices by Fourier Transform". In proc. NOC 1999, pp 317-321, 1999

[15] G. P. Agrawal, "Fiber-Optic communication systems", $3^{\text {rd }}$ ed. John Wiley \& Sons, 2010, pp. 494-497. 